$63^{\text {ème }}$ Congrès de la SFCO, 02027 (2015)

DOI:10.1051/sfco/20156302027

(C) Owned by the authors, published by EDP Sciences, 2015

\title{
COMMUNICATION
}

\section{Ostéomyélite chronique mandibulaire réfractaire : discussion thérapeutique à propos d'un cas}

\author{
Fenelon $\mathrm{M}^{*}$, Porte $\mathrm{L}^{* *}$, Lauwers $\mathrm{F}^{*}$ \\ * Service de Chirurgie maxillo faciale et plastique de la face, Place du Docteur Joseph Baylac, 31300 Toulouse \\ ** Service des Maladies infectieuses et tropicales, Place du Docteur Joseph Baylac, 31300 Toulouse
}

L'ostéomyélite est une pathologie inflammatoire de l'os. Elle s'installe dans sa partie médullaire puis progresse jusqu’à envahir le périoste de la zone concernée (Baltensperger \& Eyrich 2009). La mandibule semble être l'os le plus atteint au sein du squelette cervico facial (Prasad \& al. 2007, Theologie-Lygidakis \& al. 2011). On distingue classiquement l'ostéomyélite aigue et l'ostéomyélite chronique primitive ou secondaire (Baltensperger \& Eyrich 2009, Theologie-Lygidakis \& al. 2011). Les ostéomyélites chroniques sont souvent réfractaires aux thérapeutiques conventionnelles rendant leur prise en charge complexe (Prasad \& al. 2007).

Nous rapportons le cas d'une jeune patiente de 23 ans s'étant présentée au sein de la consultation de chirurgie Maxillo Faciale du CHU de Purpan pour une ostéomyélite mandibulaire chronique complexe. Cette ostéomyélite était survenue suite à l'avulsion des dents de sagesse et évoluait depuis deux ans. La patiente avait bénéficié au préalable d'antibiothérapies itératives associées à des curetages appuyés de la lésion ainsi qu’à une coronoïdectomie droite. Néanmoins la patiente se plaignait toujours de poussées inflammatoires douloureuses, avec un œdème jugal droit persistant et elle présentait une limitation de l'ouverture buccale à $1,5 \mathrm{~cm}$. L'imagerie par tomodensitométrie révélait une apposition périostée associée à des zones de densification médullaire hétérogènes en regard de l'angle mandibulaire et du ramus mandibulaire droits. L'IRM montrait une infiltration oedémateuse du muscle masseter étendu vers le muscle temporal et l'espace para pharyngé à droite. Il a été décidé de réaliser une décortication mandibulaire large associée à un débridement du muscle masseter, encadrés par une oxygénothérapie hyperbare ainsi qu'une antibiothérapie prolongée. La patiente est revue à 2 mois post-opératoire avec un résultat primaire satisfaisant notant une ouverture buccale à $31 \mathrm{~mm}$ en cours de rééducation et l'absence de prise d'antalgique. Elle est toujours sous antibiothérapie par Clindamycine/Amoxicilline. Un suivi à plus long terme est nécessaire.

La discussion porte sur la difficulté de prise en charge de ce type de pathologie inflammatoire. En cas d'échec de l'antibiothérapie, le recours à des anti inflammatoires, à des biphosphonate (CompyerotLacassagne 2007, Monsour \& Dalton 2010), ou encore à des séances d’oxygénothérapie hyperbare (Hudson \& al. 1993, Marx 1985) a été décrit. Une chirurgie conservatrice par curetage appuyé ou décortication y est généralement associée. Néanmoins, un taux important de récidive est parfois rapporté pouvant justifier le recours à une chirurgie interruptrice chez l’adulte (Suei \& al. 1997).

This is an Open Access article distributed under the terms of the Creative Commons Attribution License 4.0, which permits unrestricted use, distribution, and reproduction in any medium, provided the original work is properly cited. 
La décortication mandibulaire semble être une thérapeutique de choix car elle peut être facilement réitérée, elle n'expose pas le patient aux effets secondaires des thérapeutiques pharmacologiques, ni à la morbidité inhérente aux chirurgies plus agressives.

Nom et adresse du conférencier

Mathilde FENELON

Service de Chirurgie maxillo faciale et plastique de la face

Place du Docteur Joseph Baylac

31300 Toulouse (France)

mathildefenelon@live.fr 